EPJ Web of Conferences 71, 00143 (2014)

DOI: $10.1051 /$ epjconf/20147100143

(C) Owned by the authors, published by EDP Sciences, 2014

\title{
Neutron Star masses from the Field Correlator Method Equation of State
}

\author{
D. Zappalà ${ }^{1, a}$, G. F. Burgio ${ }^{1, b}$, V. Greco ${ }^{2,3, c}$, and S. Plumari ${ }^{2,3, d}$ \\ ${ }^{1}$ INFN Sezione di Catania, Via Santa Sofia 64, I-95123 Catania, Italia \\ ${ }^{2}$ Dipartimento di Fisica e Astronomia, Università di Catania, Via Santa Sofia 64, I-95123 Catania, Italia \\ ${ }^{3}$ INFN - Laboratori Nazionali del Sud, Via Santa Sofia 62, I-95125 Catania, Italia
}

\begin{abstract}
We analyse the hadron-quark phase transition in neutron stars by confronting the hadronic Equation of State (EoS) obtained according to the microscopic BruecknerHartree-Fock many body theory, with the quark matter EoS derived within the Field Correlator Method. In particular, the latter EoS is only parametrized in terms of the gluon condensate and the large distance quark-antiquark potential, so that the comparison of the results of this analysis with the most recent measurements of heavy neutron star masses provides some physical constraints on these two parameters.
\end{abstract}

\section{Introduction}

The appearance of quark matter in the interior of massive neutron stars (NS) is one of the mostly debated issues in the physics of these compact objects. If one considers only purely nucleonic degrees of freedom in the construction of the Equation of State (EoS) [1] to describe the interior of NS, it turns out that for the heaviest NS, close to the maximum mass (about two solar masses), the central particle density reaches values larger than $1 / \mathrm{fm}^{3}$, so that the nucleon cores start to touch each other, and it is hard to imagine that only nucleonic degrees of freedom can play a role. On the contrary, it can be expected that even before reaching these density values, the nucleons start to lose their identity, and quark degrees of freedom are excited at a macroscopic level.

The value of the maximum mass of NS is probably one of the physical quantities that is most sensitive to the presence of quark matter in NS. The recent observation of a large NS mass in PSR $\mathrm{J} 0348+0432$ with mass $\mathrm{M}=2.01 \pm 0.04 \mathrm{M}_{\odot}$ [2] (see also PSR J1614-2230 with mass $\mathrm{M}=1.97 \pm$ $0.04 \mathrm{M}_{\odot}$ [3]) implies that the EoS of NS matter is stiff enough to keep the maximum mass at these large values. While purely nucleonic EoS are able to accommodate such large masses [1], the presence of non-nucleonic degrees of freedom, like hyperons and quarks, tends usually to soften considerably the EoS, thus lowering the mass value, which could be incompatible with observations. The large value of the mass could then be explained only if quark matter EoS are particularly stiff. Unfortunately, the quark matter EoS is poorly known at zero temperature and at the high baryonic density appropriate for

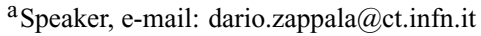

be-mail: fiorella.burgio@ct.infn.it

ce-mail: greco@lns.infn.it

de-mail: salvatore.plumari@ct.infn.it
}

This is an Open Access article distributed under the terms of the Creative Commons Attribution License 2.0, which permits unrestricted use, distribution, and reproduction in any medium, provided the original work is properly cited. 


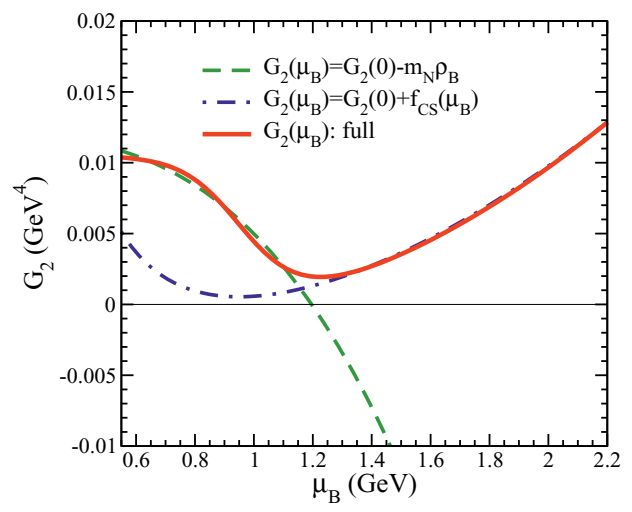

Figure 1. $G_{2}\left(\mu_{B}\right)$ as computed in [21] (dashed green) and in [24] (dot-dashed blue) with $G_{2}\left(\mu_{B}=0\right)=0.012$ $\mathrm{GeV}^{4}$. The solid red line is the effective approximation used in our analysis [5].

NS. One has, therefore, to rely on models of quark matter and compare their predictions to estimate the uncertainty of the results for the NS matter as well as for the NS structure and mass.

Here we report the main predictions, discussed in more detail in the two papers [4, 5], which are obtained with two definite nucleonic EoS developed on the basis of the nonrelativistic BruecknerHartree-Fock (BHF) many-body theory for nuclear matter and on its relativistic, Dirac-BruecknerHartree-Fock (DBHF), formulation, and by adopting, for the quark EoS, the Field Correlator Model (FCM) [6] which in principle is able to cover the full temperature-chemical potential plane. In particular, unlike the Nambu-Jona-Lasinio model, the FCM EoS contains ab initio the property of confinement, which is expected to play an important role as far as the stability of a neutron star is concerned [7]. We shall focus on the predictions of the maximum NS mass by varying the two parameters of the FCM, namely the $\bar{q} q$ potential $V_{1}$ and the gluon condensate $G_{2}$, also exploring the dependence of $G_{2}$ on the baryon chemical potential $\mu_{B}$.

In Sect. 2 we briefly recall some features of the EoS both for nuclear matter and quark matter used in this analysis, while in Sect. 3 details of the hadron-quark phase transition and related predictions for the NS masses are illustrated. The conclusions are reported in Sect. 4.

\section{EoS for nuclear and quark matter}

\subsection{Nuclear matter: the Brueckner-Bethe-Goldstone theory}

Let us briefly recall the main features of the BHF method for the nuclear matter EoS. This theoretical scheme is based on the Brueckner-Bethe-Goldstone many-body theory, which is the linked cluster expansion of the energy per nucleon of nuclear matter (see Ref.[8], chapter 1 and references therein). In this approach the bare nucleon-nucleon interaction $\mathrm{V}$ is systematically replaced by the Brueckner reaction matrix $\mathrm{G}$, which is the solution of the Bethe-Goldstone equation

$$
G(\rho ; \omega)=V+V \sum_{k_{a} k_{b}} \frac{\left|k_{a} k_{b}\right\rangle Q\left\langle k_{a} k_{b}\right|}{\omega-e\left(k_{a}\right)-e\left(k_{b}\right)} G(\rho ; \omega),
$$

where $\rho$ is the nucleon number density, $\omega$ is the starting energy, and $\left|k_{a} k_{b}\right\rangle Q\left\langle k_{a} k_{b}\right|$ is the Pauli operator. $e(k ; \rho)=k^{2} /(2 m)+U(k ; \rho)$ is the single particle energy and $U(k ; \rho)$ is the single particle potential. In 


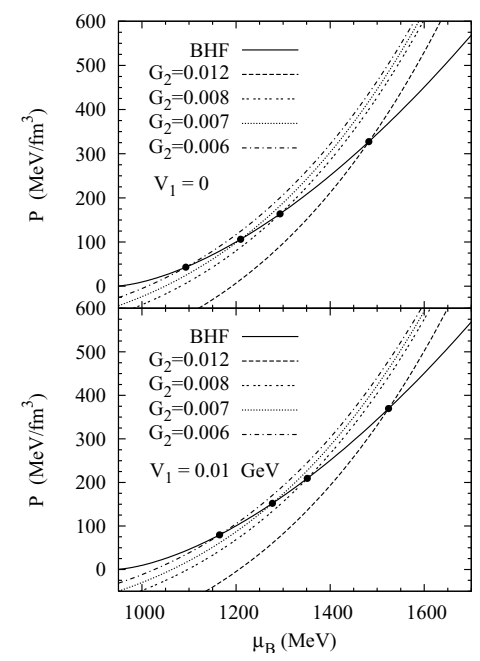

Figure 2. Pressure as a function of the baryon chemical potential $\mu_{B}$. The full line represents the BHF calculations, and the dashed ones are the pressure derived in the FCM model for several values of the ( $\mu_{B}$ independent) gluon condensate $G_{2}$ and for two different choices of the parameter $V_{1}$ (upper and lower panel).

the BHF approximation, the energy per nucleon is (the subscript " $a$ " indicates antisymmetrization of the matrix element)

$$
\frac{E}{A}(\rho)=\frac{3}{5} \frac{k_{F}^{2}}{2 m}+\frac{1}{2 A} \sum_{k, k^{\prime} \leq k_{F}}\left\langle k k^{\prime}\left|G\left(\rho ; e(k)+e\left(k^{\prime}\right)\right)\right| k k^{\prime}\right\rangle_{a}
$$

In this analysis the Argonne $v_{18}$ potential [9] is chosen as the nucleon-nucleon potential, supplemented by the so-called Urbana model [10] as three-body force. In fact, it is well known that the non-relativistic calculations, based on purely two-body interactions, fail to reproduce the correct saturation point of symmetric nuclear matter and one needs to introduce three-body forces (TBF) which, in our approach are reduced to a density dependent two-body force by averaging over the position of the third particle $[11,12]$. This allows to reproduce correctly the nuclear matter saturation point $\rho_{0} \approx 0.17 \mathrm{fm}^{-3}, E / A \approx-16 \mathrm{MeV}$, and gives values of incompressibility and symmetry energy at saturation compatible with those extracted from phenomenology [13].

Along with the BHF nonrelativistic EoS we consider its relativistic counterpart, i.e. the DBHF scheme [14] where the Bonn A potential is used for the nucleon-nucleon interaction. In the low density region $\left(\rho<0.3 \mathrm{fm}^{-3}\right)$, both BHF (including TBF) and DBHF calculations are very similar, whereas at higher densities the DBHF is slightly stiffer [1]. The discrepancy between the nonrelativistic and relativistic calculation can be easily understood by recalling that the DBHF treatment is equivalent to introducing in the nonrelativistic BHF the TBF corresponding to the excitation of a nucleon-antinucleon pair, the so-called Z-diagram [15], which is repulsive at all densities. On the contrary, in the BHF treatment with Urbana TBF, both attractive and repulsive TBF are introduced and therefore a softer EoS is expected. 


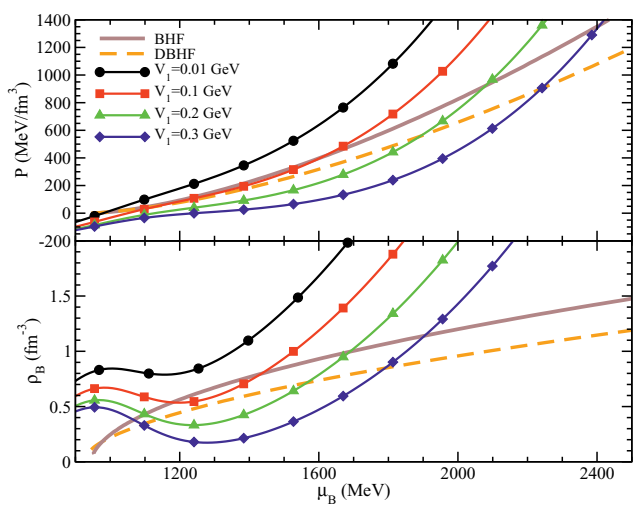

Figure 3. Pressure $P$ (upper panel) and baryon density $\rho_{B}$ (lower panel) vs. $\mu_{B}$. The quark phase EoS is computed for various $V_{1}$ and with $G_{2}\left(\mu_{B}\right)$ given by the solid red line in figure 1 . For the hadronic phase, the solid brown line and the dashed orange line respectively correspond to the BHF, and DBHF EoS.

\subsection{Quark Matter: the Field Correlator Method}

The approach based on the FCM provides a natural treatment of the dynamics of confinement in terms of the Color Electric $\left(D^{E}\right.$ and $\left.D_{1}^{E}\right)$ and Color Magnetic $\left(D^{H}\right.$ and $D_{1}^{H}$ ) Gaussian correlators, being the former one directly related to confinement, so that its vanishing above the critical temperature implies deconfinement [6]. The extension of the FCM to finite temperature $T$ and chemical potential $\mu_{q}=0$ gives analytical results in reasonable agreement with lattice data, thus allowing to describe correctly the deconfinement phase transition [16-18]. In order to derive an EoS of the quark-gluon matter in the range of baryon density typical of the neutron star interiors, we have to extend the FCM to finite values of the chemical potential $[16,17]$. In this case, the quark pressure for a single flavour is simply given by [16-18]

$$
P_{q} / T^{4}=\frac{1}{\pi^{2}}\left[\phi_{\nu}\left(\frac{\mu_{q}-V_{1} / 2}{T}\right)+\phi_{v}\left(-\frac{\mu_{q}+V_{1} / 2}{T}\right)\right]
$$

where $\phi_{v}(a)=\int_{0}^{\infty} d u\left(u^{4} / \sqrt{u^{2}+v^{2}}\right)\left(\exp \left[\sqrt{u^{2}+v^{2}}-a\right]+1\right)^{-1}$ with $v=m_{q} / T$, and $V_{1}$ is the large distance static $\bar{q} q$ potential which, in our analysis, is treated as a free parameter.

The gluon contibution to the pressure is

$$
P_{g} / T^{4}=\frac{8}{3 \pi^{2}} \int_{0}^{\infty} d \chi \chi^{3} \frac{1}{\exp \left(\chi+\frac{9 V_{1}}{8 T}\right)-1}
$$

and the total pressure, that corresponds to the EoS in this phase, is

$$
P_{q g}=\sum_{j=u, d, s} P_{q}^{j}+P_{g}-\frac{\left(11-\frac{2}{3} N_{f}\right)}{32} \frac{G_{2}}{2}
$$

where $P_{q}^{j}$ and $P_{g}$ are respectively given in Eq. (3) and (4), and $N_{f}$ is the flavour number. The last term in Eq. (5) corresponds to the difference of the vacuum energy density in the two phases and $G_{2}$ is the gluon condensate whose numerical value, determined by the QCD sum rules at zero temperature and chemical potential, is known with large uncertainty: $G_{2}=0.012 \pm 0.006 \mathrm{GeV}^{4}$. 


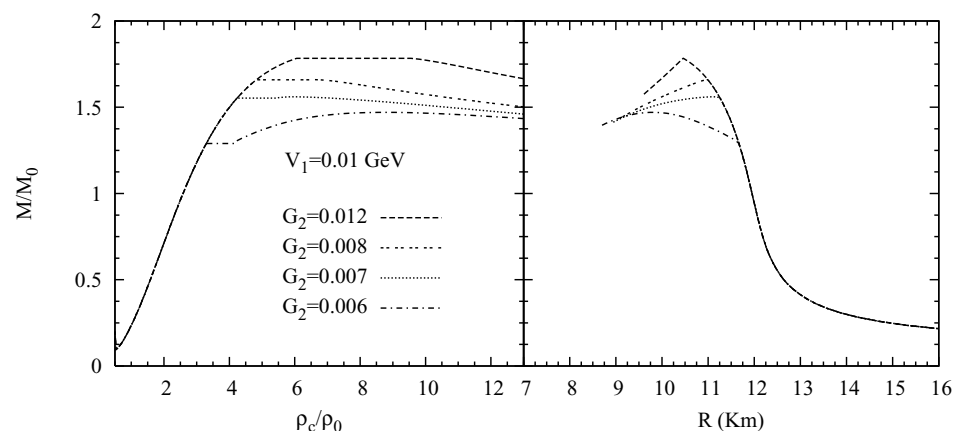

Figure 4. The NS mass (in units of the solar mass) vs. the central baryon density (left panel) and the corresponding radius (right panel), for $V_{1}=0.01 \mathrm{GeV}$ and some values of $\mu_{B}$ independent $G_{2}$.

Therefore the EoS in Eq.(5) essentially depends on two parameters, namely the quark-antiquark potential $V_{1}$ and the gluon condensate $G_{2}$. In addition, at finite temperature and vanishing baryon density, a comparison with the recent available lattice calculations provides clear indications about the specific values of these two parameters, and in particular their values at the critical temperature $T_{c}$. These estimates can be related to the corresponding values of the parameters at $T=\mu_{B}=0$ and, in particular, one finds $V_{1}\left(T=\mu_{B}=0\right)=0.8 \div 0.9 \mathrm{GeV}[5,19]$. In our analysis, we are concerned about the dependence of $V_{1}$ and $G_{2}$ on the baryon chemical potential $\mu_{B}$ and, due to the absence of theoretical indications on $V_{1}$, except for some lattice simulations that suggest no dependence of $V_{1}$ on $\mu_{B}$, at least for very small $\mu_{B}[17,20]$, we treat $V_{1}$ as a free parameter with no dependence on $\mu_{B}$.

On the other hand, there are indications about the dependence of the gluon condensate $G_{2}$ on $\mu_{B}$. In particular, the QCD sum rules technique has been used to study some hadronic properties within a nuclear matter environment at $T=0$ [21-23], and it has been found that the gluon condensate decreases linearly with the baryon density $\rho_{B}$, with small nonlinear corrections that can be neglected for our purposes. The corresponding curve is the dashed green line plotted in figure 1. According to this decreasing trend, the gluon condensate vanishes at some value of the baryon density and one expects that a transition to the deconfined state should occur before reaching this point.

A different analysis is presented in $[24,25]$ where $G_{2}\left(\mu_{B}\right)$ is computed in two-color $\left(N_{c}=2\right)$ QCD, where many technical problems that affect the theory with $N_{c}=3$ are absent. In particular, the difference $f_{C S}(\mu)=G_{2}(\mu)-G_{2}(0)$, which is computed from the energy momentum tensor of an effective chiral lagrangian, shows an initial decrease which, after reaching a minimum, is followed by a continuous growth, and this trend is explained with the appearance of a weakly interacting gas of diquarks. In $[24,25]$, it is then suggested that $G_{2}\left(\mu_{B}\right)$ in full three-color $\left(N_{c}=3\right)$ QCD has the same qualitative behavior of the corresponding variable in two-color $\left(N_{c}=2\right) \mathrm{QCD}$, and therefore the plot of $f_{C S}(\mu)$, with the proper choice of the parameters for $N_{c}=3$ and fixing $G_{2}\left(\mu_{B}=0\right)=0.012 \mathrm{GeV}^{4}$, is reported in figure 1 as the dot-dashed blue curve.

Then, by following the first indication at low $\mu_{B}$ and the second one at larger $\mu_{B}$, in our analysis we assumed a chemical potential dependent gluon condensate given by the solid red line in figure 1, which starts at $G_{2}\left(\mu_{B}=0\right)=0.012 \mathrm{GeV}^{4}$ and approximates the dashed green curve at small $\mu_{B}$ and the dot-dashed blue one after the crossing of these two curves. The analytic form of $G_{2}\left(\mu_{B}\right)$ is chosen to avoid unphysical features due to possible discontinuities in its derivatives. Along with this particular choice of $G_{2}\left(\mu_{B}\right)$, we shall also display the results obtained with $\mu_{B}$ independent $G_{2}$. 


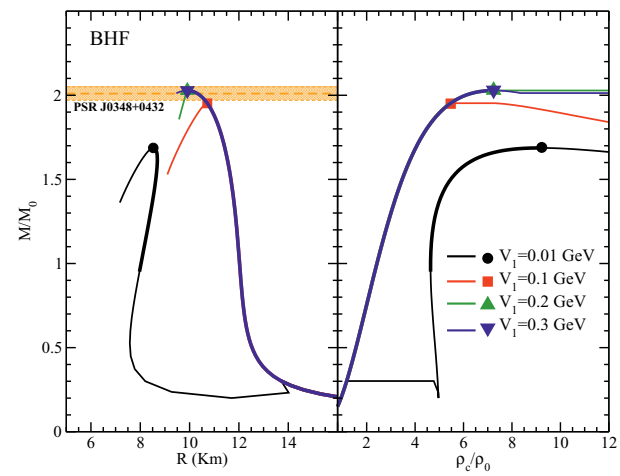

Figure 5. The mass-radius (left panel) and the mass-central density relation (right panel) for the BHF hadronic EoS and with $V_{1}$ and $G_{2}\left(\mu_{B}\right)$ as in figure 3. The full symbols denote the value of the maximum mass. Stable configurations are displayed by thick lines, whereas thin lines indicate unstable configurations.

\section{Hadron-quark phase transition and the corresponding NS masses}

The transiton between the hadron and quark phase is described by comparing the pressure of the two phases. We adopt the simple Maxwell construction, by assuming a first order hadron-quark phase transition and by imposing thermal, chemical, and mechanical equilibrium between the two phases. This implies that the phase coexistence is determined by a crossing point in the pressure vs. chemical potential plot, as shown in figures 2 and 3. In figure 2 the pressure $P$ is displayed as function of the baryon chemical potential $\mu_{B}$ in the nonrelativistic BHF case for the hadronic phase, and at very small $V_{1}$ and $G_{2}$ taken independent of $\mu_{B}$ for the quark phase. Instead, figure 3 shows the pressure $P$ (upper panel) and the baryon density, $\rho_{B}=\partial P / \partial \mu_{B}$, (lower panel) vs. $\mu_{B}$ for the hadronic EoS's (solid brown line for BHF and dashed orange line for DBHF), whereas symbols are the results for quark matter EoS in the FCM and different choices of $V_{1}$ and $\mu_{B}$ dependent $G_{2}\left(\mu_{B}\right)$, as introduced at the end of Sect. 2.2.

We observe that, both with increasing $G_{2}$ as in figure 2 and $V_{1}$ as in figure 3, the phase transition point is shifted to larger chemical potentials which, in principle, indicates that the NS possesses a thicker hadronic layer for larger values of these parameters. In addition, while in figure 2 the derivative of the pressure in the quark phase always grows with $\mu_{B}$, in figure 3 there is a region where the derivative of the pressure, $\rho_{B}$, decreases (see lower panel). This behavior, as it is evident from a comparison of figures 2 and 3 , is due to the particular parametric form of the gluon condensate $G_{2}\left(\mu_{B}\right)$ shown in figure 1 and, as shown below, it is the source of unstable neutron stars configurations.

The EoS is the fundamental input for solving the well-known hydrostatic equilibrium equations of Tolman, Oppenheimer, and Volkoff [26] for the pressure $P$ and the enclosed mass $m$

$$
\begin{gathered}
\frac{d P(r)}{d r}=-\frac{G m(r) \epsilon(r)}{r^{2}}\left[1+\frac{P(r)}{\epsilon(r)}\right]\left[1+\frac{4 \pi r^{3} P(r)}{m(r)}\right]\left[1-\frac{2 G m(r)}{r}\right]^{-1} \\
\frac{d m(r)}{d r}=4 \pi r^{2} \epsilon(r)
\end{gathered}
$$

being $\epsilon$ the total energy density ( $G$ is the gravitational constant). For a chosen central value of the energy density $\rho_{c}$, the numerical integration of Eqs. (6) and (7) provides the mass-radius relation. 


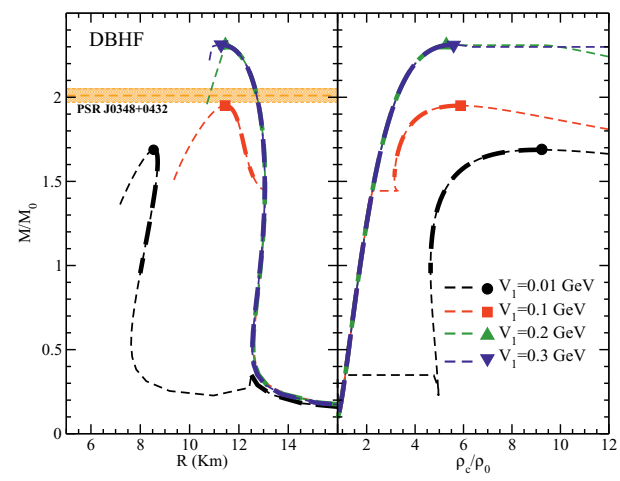

Figure 6. Same as in figure 5 but with the DBHF EoS used for the hadronic phase.

We first consider the parametrization analyzed in the lower panel of figure 2 and we display in figure 4 the corresponding gravitational mass (in units of solar mass $\mathrm{M}_{\odot}=2 \times 10^{33} \mathrm{~g}$ ) as a function of the central baryon density $\rho_{c}$, normalized with respect to the saturation value $\rho_{0}=0.17 \mathrm{fm}^{-3}$, (left panel) and of the NS radius R (right panel). We observe that the maximum mass of the star is an increasing function of $G_{2}$ although its value is lower than the highest experimentally observed mass and therefore higher inputs for $V_{1}$ are required. However we recall that gravitationally stable NS configurations require $\mathrm{dM} / \mathrm{d} \rho_{c}>0$ [26] and then, only those two curves with lower maximum mass in figure 4 admit stable configurations with an inner quark matter core.

Finally we consider the more complex case with $\mu_{B}$ dependent gluon condensate, presented in figure 3. The corresponding NS masses are displayed in figures 5 and 6 , respectively for the nonrelativistic BHF and relativistic DBHF hadronic EoS. The orange band represents the recently observed neutron star PSR J0348+0432 [2]. In this figures we have marked the gravitationally stable configurations by thick lines, whereas full symbols denote the maximum mass. Unstable configurations are instead displayed by thin lines. Among the unstable configurations, we signal those characterized by increasing mass and decreasing central density, which are related to the nonmonotonic behavior of the derivative of the pressure in the quark phase in figure 3, as anticipated above.

For the hadronic BHF EoS in figure 5 the observational data require $V_{1}>0.1 \mathrm{GeV}$ and, for $V_{1}>0.2 \mathrm{GeV}$ the maximum mass does not grow beyond 2.03 solar masses [5]. In addition, in this range of $V_{1}$, the maximum mass corresponds to the intersection of the hadronic and quark branches, while stable configurations with a quark matter inner core are realized only for $V_{1}<0.095 \mathrm{GeV}$, but with values of the maximum mass that are incompatible with the current observational data.

The stiffer structure of the hadronic DBHF EoS yields larger NS masses as shown in figure 6 . The qualitative picture is similar to that of figure 5 , but this time at $V_{1}=0.1 \mathrm{GeV}$ we find stable NS with maximum mass compatible with observations and with a quark matter content. We also find that for $V_{1}$ larger than $\mathrm{V}_{1} \approx 0.12 \mathrm{GeV}$ the quark core disappears and for $V_{1}>0.2 \mathrm{GeV}$ the maximum mass stays at its highest value of 2.31 solar masses.

\section{Conclusions}

The study of the maximum mass of NS computed according to the FCM description of quark matter and to the nonrelativistic BHF or relativistic DBHF EoS of hadronic matter, provides some indications concerning the parameters of the FCM when the results are compared with the recently observed NS 
masses. Observational data require stiff EoS and in fact the smoother hadronic BHF EoS does not allow NS heavier than about 2 solar masses, which is still in agreement with the observational data, while the DBHF EoS reaches 2.31 solar masses. In any case such large mass configurations can be reached only by increasing the two parameters $V_{1}$ and $G_{2}$ of the FCM, because for small values of these parameters the typical maximum mass is well below the observational limit. However, while for small $V_{1}$ and $G_{2}$, stable configurations with an inner core of quark matter are predicted, for those higher values of $V_{1}$ and $G_{2}$ that predict sufficiently heavy masses, the quark core disappears and the star has only hadronic content. The only small window that predicts sufficiently heavy stable stars with a quark matter core is given by the DBHF EoS combined with the FCM for $0.1 \mathrm{GeV} \lesssim V_{1} \lesssim 0.12 \mathrm{GeV}$ and $G_{2}(\mu)$ parametrized as in Sect. 2.2.

Finally, it must be noticed that the relevant range of $V_{1}$ found in this analysis $(0.1 \div 0.2 \mathrm{GeV})$, is rather different from the one obtained from the FCM analysis of the transition at finite temperature $(0.8 \div 0.9 \mathrm{GeV})$. In [5] it has been suggested that this difference could be an indication that, at finite density and vanishing temperature, the quark-antiquark potential $V_{1}$ is to be replaced by a quark-quark effective interaction whose strength is about $V_{1} / 4$.

\section{References}

[1] G. Taranto, M. Baldo, and G.F. Burgio, Phys. Rev. C 87, 045803 (2013).

[2] J. Antoniadis et al., Science 340, 6131 (2013).

[3] P. Demorest et al., Nature 467, 1081 (2010).

[4] M. Baldo, G.F. Burgio, P. Castorina, S. Plumari, and D. Zappalà, Phys. Rev. D 78, 063009 (2008).

[5] S. Plumari, G.F. Burgio, V. Greco and D. Zappalà, Phys. Rev. D 88, 083005 (2013) .

[6] A. Di Giacomo, H.G. Dosch, V.I. Shevchenko, and Y.A. Simonov, Phys. Rep 372, 319 (2002) .

[7] M. Baldo, G.F. Burgio, P. Castorina, S. Plumari, D. Zappalà, Phys. Rev. C 75, 035804 (2007) .

[8] M. Baldo, Nuclear Methods and the Nuclear Equation of State - International Review of Nuclear Physics, Vol. 8 (World Scientific, Singapore, 1999).

[9] R.B. Wiringa, V.G.J. Stoks, and R. Schiavilla, Phys. Rev. C 51, 38 (1995).

[10] J. Carlson, V.R. Pandharipande, and R.B. Wiringa, Nucl. Phys. A 401, 59 (1983).

[11] M. Baldo, I. Bombaci, and G.F. Burgio, Astron. Astrophys. 328, 274 (1997).

[12] X.R. Zhou, G.F. Burgio, U. Lombardo, H.-J. Schulze, W. Zuo, Phys. Rev. C 69, 018801 (2004).

[13] W. D. Myers and W. J. Swiatecki, Nucl. Phys. A 601, 141 (1996); Phys. Rev. C 57, 3020 (1998).

[14] T. Gross-Boelting, C. Fuchs, and A. Faessler, Nucl. Phys. A 648, 105 (1999).

[15] G. E. Brown, W. Weise, G. Baym, and J. Speth, Comments Nucl. Part. Phys. 17, 39 (1987).

[16] Yu.A. Simonov, and M.A. Trusov, JETP Lett. 85, 598 (2007).

[17] Yu.A. Simonov, and M.A. Trusov, Phys. Lett. B 650, 36 (2007) .

[18] A. V. Nefediev, Yu.A. Simonov, and M.A. Trusov, Int. J. Mod. Phys. E 18, 549 (2009).

[19] I. Bombaci, and D. Logoteta, MNRAS 433, L79 (2013).

[20] M. Doring, S. Ejiri, O. Kaczmarek, F. Karsch, and E. Laermann, Eur. Phys. J. C 46, 179 (2006).

[21] T. D. Cohen, R. J. Furnstahl, and D. K. Griegel, Phys. Rev. C 45, 1881 (1992).

[22] E. G. Drukarev, M. G. Ryskin, and V. A. Sadovnikova, Prog. Part. Nucl. Phys. 47, 73 (2001).

[23] M. Baldo, P. Castorina, and D. Zappalà, Nucl. Phys. A 743, 3 (2004).

[24] M. A. Metlitski, and A. R. Zhitnitsky, Nucl. Phys. B 731, 309 (2005).

[25] A. R. Zhitnitsky, AIP Conf.Proc. 892, 518 (2007).

[26] S.L. Shapiro and S.A. Teukolsky, Black Holes, White Dwarfs and Neutron Stars (John Wiley and Sons, New York, 1983). 